\title{
Eigenvector perturbation methodology for uncertainty quantification of turbulence models
}

\author{
Roney L. Thompson \\ Department of Mechanical Engineering, Federal University of Rio de Janeiro, Brazil \\ Aashwin Ananda Mishra and Gianluca Iaccarino \\ Center for Turbulence Research, Stanford University, Stanford, California 94305, USA \\ Wouter Edeling \\ Centrum Wiskunde \& Informatica, Amsterdam, The Netherlands \\ Luiz Sampaio \\ Department of Energy Resources Engineering, Stanford University, Stanford, California 94305, USA
}

(Received 6 November 2018; published 9 April 2019)

\begin{abstract}
Reynolds-averaged Navier-Stokes (RANS) models are the primary numerical recourse to investigate complex engineering turbulent flows in industrial applications. However, to establish RANS models as reliable design tools, it is essential to provide estimates for the uncertainty in their predictions. In the recent past, an uncertainty estimation framework relying on eigenvalue and eigenvector perturbations to the modeled Reynolds stress tensor has been widely applied with satisfactory results. However, the methodology for the eigenvector perturbations is not well established. Evaluations using only eigenvalue perturbations do not provide comprehensive estimates of model form uncertainty, especially in flows with streamline curvature, recirculation, or flow separation. In this article, we outline a methodology for the eigenvector perturbations using a predictor-corrector approach, which uses the incipient eigenvalue perturbations along with the Reynolds stress transport equations to determine the eigenvector perturbations. This approach was applied to benchmark cases of complex turbulent flows. The uncertainty intervals estimated using the proposed framework exhibited substantial improvement over eigenvalue-only perturbations and are able to account for a significant proportion of the discrepancy between RANS predictions and high-fidelity data.
\end{abstract}

DOI: 10.1103/PhysRevFluids.4.044603

\section{INTRODUCTION AND OVERVIEW}

Turbulent flows play an important role in a wide number of engineering design problems. Due to the dissimilar nature of such flows, the predictive methods used to study them must be robust and applicable for a range of flows, yet possess a high degree of accuracy. As the processes of design involves repeated iterations, the predictive methods must also be computationally economical. Accordingly, even with the increasing developments in computational hardware and the use of new techniques that enable significant progress on high-fidelity simulations of turbulent flows using direct numerical simulations or large eddy simulations, Reynolds-averaged Navier-Stokes (RANS) models are still the predominant recourse employed to investigate turbulence problems in the industry.

In spite of their widespread use, RANS models have many deficiencies. The assumptions and simplifications made during the formulation of such RANS closures can limit their ability to account 
for fundamental turbulence processes and specific flow phenomena. Such simplifications include the use of the eddy viscosity hypothesis to relate Reynolds stresses to the mean rate of strain; the use of the gradient-diffusion hypothesis to model turbulent transport, the use of an approximate evolution equation for the dissipation process. Additionally, RANS models as single-point closures have inherent limitations in representing nonlocal physics [1], such as due to the pressure-strain correlation. While relatively accurate in simpler flows, these assumptions can be a major source of discrepancy in complex turbulent flows. For instance, in turbulent flows with significant effects of mean rotation, such as swirl or strong streamline curvature, the fidelity of linear eddy-viscositybased closures is often unsatisfactory [2]. In a congruous vein, the performance of two-equation models is found to be erroneous for cases with noninertial frames of reference [3]. In turbulent flows with flow separation and reattachment, eddy-viscosity-based models have had limited success $[4,5]$. In turbulent flows through noncircular channels, isotropic eddy-viscosity-based models are not able to reproduce the secondary flows that develop near the corners of the domain [6]. In this light, to aid in the establishment of RANS simulations as reliable tools for the engineering design process, there is need for quantifying the uncertainty in model predictions.

There have been many approaches developed to estimate the uncertainties in RANS model predictions. For instance, one of the recourses utilizes data driven approaches, with machinelearning algorithms to estimate RANS uncertainties. Singh and Duraisamy [7] and Parish and Duraisamy [8] utilize such a methodology where full-field datasets are used to calibrate functional forms of model discrepancies and develop model corrections. Ling and Templeton [9] use a data-driven approach with different machine-learning tools to identify flow zones with high degrees of model form uncertainty.

In contrast to such data driven approaches, a methodology developed by Iaccarino and coworkers [10,11] presents a purely physics-based approach to estimate the model-form uncertainties. This framework approximates uncertainties using perturbations to the modeled Reynolds stress eigenvalues, eigenvectors and the turbulent kinetic energy. This formulation has been applied to a wide range of engineering problems with significant success [12-15]. At the interface of these paradigms, investigators have used this physics-based approach guided by data driven techniques to ascertain uncertainty. For instance, Xiao et al. [16] and Wu et al. [17] have used a data-driven framework to determine the requisite perturbations to the Reynolds stress eigenvalues and eigenvectors for different flows to ascertain model form uncertainties. Xiao et al. [18] proposed using Euler angles for parametrizing perturbations on Reynolds stress eigenvectors. Wu et al. [19], however, used another parametrization scheme for perturbations of the eigenvectors based on unit quaternion. Wu et al. [20] showed that the unit-quaternion-based parametrization of the eigenvector rotation is superior to the Euler-angle-based approach in the context of machine-learning-assisted RANS modeling and UQ. Wang et al. [21] compared random matrix-based approaches to physics-based approaches for introducing such perturbations.

The perturbation approach to estimating the structural uncertainty in RANS models uses sequential perturbations to the Reynolds stress eigenvalues, eigenvectors and the turbulent kinetic energy. The methodology of perturbing the Reynolds stress eigenvalues is well accepted and involves sampling from the extremal states of the Reynolds stress componentiality. This ensures that the perturbations account for as much of the discrepancy in the Reynolds stress eigenvalues, while still remaining realizable. The methodology for eigenvector perturbations is, however, not universally accepted. Wang et al. [21] first attempted to perturb the Reynolds stress eigenvectors by using Euler angles to fully explore uncertainty space in RANS simulations. Wang et al. [22] then attempted to learn the functional form of discrepancies in Euler angles based on machine-learning techniques. However, such approaches, that use machine-learning algorithms to supplant the physics-based framework, may be limited by the availability of adequately large and pertinent high-fidelity data sets to learn from. Alternatively, Iaccarino et al. [11] developed a purely physics-based eigenvector perturbation methodology that attempts to estimate the RANS model-form uncertainty using modulation of the turbulence production process. This methodology explores the extremal alignments of the Reynolds stress eigenvectors with the mean rate of strain 
eigen-directions. However, due to its inherent use of only the extremal alignments, such an approach may lead to conservative estimates for the turbulence model uncertainty bounds, wherein the bounds over-predict the magnitude of the RANS discrepancy. In this vein, an alternate methodology for estimating the eigenvector perturbations may be beneficial for uncertainty estimation. Ideally, the methodology should ascertain the extent of eigenvector perturbations by constraining them. This would ensure that the uncertainty estimates are not needlessly large. Furthermore, it is generally accepted that the RANS model uncertainty is not uniform in space. Consequently, the perturbations that seek to account for this uncertainty should be varying in space as well.

The need for such a methodology is underscored by the importance of eigenvector perturbations in uncertainty quantification. The perturbations to the eigenvectors are critically important as the misalignment between the mean rate of strain and the Reynolds stresses is a major source of discrepancy for RANS models, especially in flows with significant streamline curvature, recirculation, flow separation, reattachment, etc. This includes turbulent flows over curved surfaces, behind bluff bodies, in jet efflux, etc.

In this article, we propose such an alternate methodology for the eigenvector perturbations using a predictor-corrector approach, that uses eigenvalue perturbations along with the Reynolds stress transport equations to determine the eigenvector perturbations. This is applied to benchmark cases of complex turbulent flow and the results are compared with eigenvalue-only perturbations to quantify the improvements in uncertainty estimates.

\section{MATHEMATICAL FRAMEWORK AND DETAILS}

The overarching objective of this investigation is to generate interval estimates for different quantities of interest in turbulence simulations. These interval estimates should account for the structural uncertainty in turbulence models. To this end, we utilize a physics-based approach and stochastic descriptions of the uncertainties is not used.

\section{A. Eigenvector perturbations: Methodology}

Utilizing the eigendecomposition (spectral decomposition), the Reynolds stress tensor, $\mathbf{R}=$ $\left\langle u_{i} u_{j}\right\rangle$, can be expressed as

$$
\mathbf{R}=2 k\left(\frac{1}{3} \mathbf{I}+\mathbf{Q} \mathbf{\Lambda} \mathbf{Q}^{\mathbf{T}}\right),
$$

where $\mathbf{I}$ is the identity tensor, $\mathbf{Q}$ is the orthonormal matrix of the unit eigenvectors of $\mathbf{b}$, the Reynolds stress anisotropy tensor $\left(\mathbf{b}=\frac{\mathbf{R}}{2 k}-\frac{\delta}{3}\right.$, where $\delta$ represents the Kronecker $\delta$ tensor $)$, and $\boldsymbol{\Lambda}$ is the diagonal matrix of the eigenvalues of $\mathbf{b}$. Here, the symmetry of $\mathbf{R}$ allows the transpose to be adopted rather than the inverse. The limitations of classical turbulence models can be re-expressed using this decomposition. For instance, one of the key ramifications of the eddy-viscosity hypothesis is that it obligates the modeled Reynolds stress to share its eigen-directions with the mean rate of strain tensor. Consequently, the eigenvectors of the modeled Reynolds stresses are coincident with those of the mean rate of strain. While this is true in simple shear flows, it is of limited accuracy in complex engineering flows [2,3]. Similarly, assumptions made in the gradient-diffusion hypothesis lead to imperfect representation of the amplitude of the Reynolds stress ellipsoid [11] and the form of the eddy-viscosity hypothesis leads to unsatisfactory expression for the Reynolds stress anisotropy eigenvalues [10].

To account for the errors due to such closure assumptions, this spectral representation of the Reynolds stress tensor is perturbed. These perturbations are injected directly into the modeled Reynolds stress during the CFD solution iterations. This perturbed form of the Reynolds stress $\mathbf{R}^{*}$ is expressed as

$$
\mathbf{R}^{*}=2 k^{*}\left(\frac{1}{3} \mathbf{I}+\mathbf{Q}^{*} \boldsymbol{\Lambda}^{*} \mathbf{Q}^{\mathbf{T} *}\right),
$$


where ${ }^{*}$ represents the perturbed quantities. The perturbations to the eigenvalues, $\boldsymbol{\Lambda}$, correspond to varying the componentiality of the flow (or the shape of the Reynolds stress ellipsoid). Similarly, the perturbations to the eigenvectors vary the orientation of the Reynolds stress ellipsoid with respect to the eigen-directions of the mean rate of strain. Additionally, by changing this relative alignment, the eigenvector perturbations modulate the turbulence production process, further underscoring their importance.

The eigenvector perturbation methodology outlined in this investigation is agnostic to the specific method used to perturb Reynolds stress eigenvalues. This procedure consists of a sequential twostage approach in which, after the perturbation of the eigenvalues is injected, the corresponding perturbation of the eigenvectors is introduced.

To determine the eigenvector perturbations, we utilize the mean momentum equations to obtain intermediate values of the mean velocity field. This is used in the Reynolds Stress Transport equations to deduce the corresponding values of the eigenvector perturbations. These eigenvector perturbations, along with the initial eigenvalue perturbations, are used to re-constitute the perturbed Reynolds stress tensor. Thence, this final form of the Reynolds stresses are used to update the mean velocity field using the mean momentum equations. Eddy-viscosity-based closures do not enforce an explicit relationship between the eigenvalues and eigenvectors of the modeled Reynolds stress tensor. However, the Reynolds Stress Transport equations explicitly solve transport equations for the components of the Reynolds stress. Thus, they lead to a natural correspondence between any changes to the eigenvalues of the modeled Reynolds stress with the corresponding changes to the eigenvectors. The explicit form of the Reynolds Stress Transport equation is

$$
\frac{\bar{D} \mathbf{R}}{\bar{D} t}=-\nabla \mathbf{v} \cdot \mathbf{R}-\mathbf{R} \cdot \nabla^{T} \mathbf{v}+v \nabla^{2} \mathbf{R}+\mathbf{\Gamma},
$$

where $\frac{\bar{D}}{\bar{D} t}$ represents the mean substantial derivative and the tensor $\Gamma$ accounts for the sum of all tensors that need to be modeled, explicitly,

$$
\Gamma=\nabla \cdot \mathfrak{C}+\Pi-\boldsymbol{\epsilon},
$$

where $\mathfrak{C}$ is the triple correlation of the velocity fluctuations, $\Pi$ is the velocity-pressure-gradient tensor, and $\epsilon$ is the dissipation tensor. To apply the eigenvector perturbation procedure, we compute the corresponding evolution for the Reynolds stress anisotropy tensor. The transport equation for $\mathbf{b}$ is given by

$$
\frac{\bar{D} \mathbf{b}}{\bar{D} t}=-\nabla \mathbf{v} \cdot \mathbf{b}-\mathbf{b} \cdot \nabla^{T} \mathbf{v}+\frac{2}{3} \mathbf{b}: \mathbf{S}+v \nabla^{2} \mathbf{b}+\hat{\boldsymbol{\Gamma}}
$$

where $\hat{\boldsymbol{\Gamma}} \equiv \boldsymbol{\Gamma}-\frac{1}{3} \operatorname{tr} \boldsymbol{\Gamma}+\frac{4}{3} \mathbf{S}$. We represent the Reynolds stress anisotropy and the mean velocity from the unperturbed RANS simulations via $\mathbf{b}_{a}$ and $\mathbf{v}$. The mean momentum equation is given by

$$
\frac{\bar{D} \mathbf{v}}{\bar{D} t}=v \nabla^{2} \mathbf{v}-\frac{1}{\rho} \nabla \mathbf{P}-\nabla \mathbf{R} .
$$

Our procedure can be summarized using the sequential steps:

(1) Use a standard procedure that perturbs the eigenvalues of $\mathbf{b}$ to obtain a range of variability of the Reynolds Stress anisotropy tensor, $\Delta \mathbf{b}_{\text {eval }}$. Obtain the intermediate Reynolds stress anisotropy as $\mathbf{b}_{\text {int }}=\mathbf{b}_{a}+\Delta \mathbf{b}_{\text {eval }}$.

(2) Compute $\hat{\boldsymbol{\Gamma}}=\frac{\bar{D} \mathbf{b}_{a}}{\bar{D} t}+\nabla \mathbf{v} \cdot \mathbf{b}_{a}+\mathbf{b}_{a} \cdot \nabla^{T} \mathbf{v}-v \nabla^{2} \mathbf{b}_{a}$ from Eq. (5) using the modeled Reynolds stress field, $R_{\text {mod }}$.

(3) Update the mean velocity field by solving the momentum equation using the intermediate Reynolds stress tensor, $\mathbf{R}_{\text {int }}$.

(4) Use the Reynolds Stress Transport equation with $\hat{\Gamma}$ to find a new perturbed Reynolds stress tensor, $\mathbf{b}_{1}$.

(5) Extract the corresponding eigenvector perturbation, $\Delta \mathbf{b}_{\text {evec }}$, with respect to $\mathbf{b}_{a}$. 

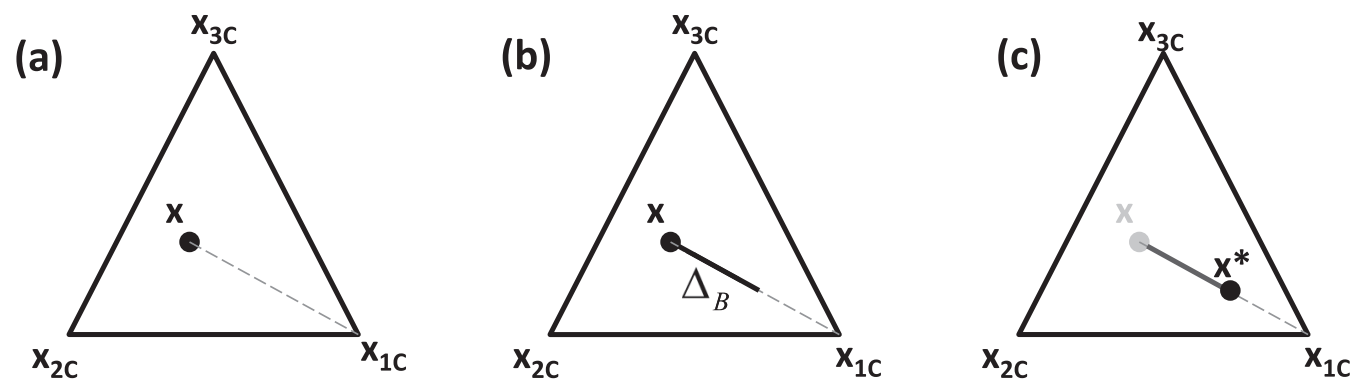

FIG. 1. Schematic outline of the eigenvalue perturbations on the barycentric triangle, starting from an arbitrary state.

(6) Find the new Reynolds stress tensor, $\mathbf{b}^{*}=\mathbf{b}_{a}+\Delta \mathbf{b}_{\text {eval }}+\Delta \mathbf{b}_{\text {evec }}$.

(7) Solve the mean momentum equation with $\mathbf{b}^{*}$ to recompute the final mean velocity field.

\section{B. Eigenvalue perturbations}

In this section, we outline the eigenvalue perturbation methodology used in conjunction with the eigenvector perturbations. For simplicity, we will use the spatially uniform eigenvalue perturbation method described in Emory et al. [10]. Given the eigenvalues of a baseline eddy-viscosity model $\left(\Lambda^{(\mathrm{bl})}\right)$, the perturbed eigenvalues $\Lambda^{*}$ are obtained by perturbing an amount $\Delta_{B}$ toward the limiting states of Reynolds stress componentiality. Mathematically, this reads

$$
\Lambda^{*}=\Lambda^{(\mathrm{bl})}+\Delta_{B}\left(\Lambda^{(\alpha c)}-\Lambda^{(\mathrm{bl})}\right), \quad \alpha=1,2,3 .
$$

Here, $\Lambda^{(\alpha c)}$ is the selected limiting state, i.e., one, two, or three-component turbulence. These are a priori known states, given by $\Lambda^{(1 c)}:=\operatorname{diag}(2 / 3,-1 / 3,-1 / 3), \Lambda^{(2 c)}:=\operatorname{diag}(1 / 6,1 / 6,-1 / 3)$ and $\Lambda^{(3 c)}:=\operatorname{diag}(0,0,0)$, the latter corresponding to isotropic turbulence. The perturbation magnitude is a user-specified amount $\Delta_{B} \in[0,1]$. This eigenvalue perturbation is schematically outlined in Fig. 1 using the barycentric map developed by Banerjee et al. [23]. Using barycentric coordinates ensures that the final perturbed state of the Reynolds stresses remains insode the barycentric triangle and thus, is realizable. The perturbations are aligned towards the vertices of the barycentric triangle (or the limiting states of turbulence), as shown in Fig. 1. The magnitude of the eigenvalue perturbation in the barycentric triangle is represented by $\Delta_{B} \in[0,1]$, schematically illustrated in Fig. 1(b). The perturbed barycentric coordinates $\mathbf{x}^{*}$ are given by $\mathbf{x}^{*}=\mathbf{x}+\Delta_{B}\left(\mathbf{x}^{\mathbf{t}}-\mathbf{x}\right)$, where $\mathbf{x}^{\mathbf{t}}$ denotes the target vertex (representing one of the one-, two-, or three-component limiting states) and $\mathbf{x}$ is the unperturbed model prediction. Thus, $\Delta_{B}=0$ would leave the state unperturbed and $\Delta_{B}=1$ would perturb any arbitrary state out to the vertices of the barycentric triangle. In Fig. 1, this eigenvalue perturbation methodology is illustrated, starting from an arbitrary Reynolds stress componentiality. For this illustration, the direction of the perturbation $\mathbf{x}^{\mathbf{t}}$ is chosen toward $\mathbf{x}_{1 C}$ and the magnitude of perturbation $\Delta_{B}$ is chosen as 0.5 . The initial $\mathbf{x}$ and perturbed $\mathbf{x}^{*}$ states are exhibited in the figure, along with the transition between the unperturbed to the perturbed state.

Thus, in this approach, the eigenvalue perturbations are carried out using the Barycentric map and the eigenvector perturbations, using guidance from the Reynolds stress transport equations. While perturbations to the turbulent kinetic energy may also be incorporated, no direct perturbations to the turbulent kinetic energy are incorporated in the results, along the lines of prior researchers $[10,11]$.

\section{ERRORS ASSOCIATED WITH BOUSSINESQ AND NONLINEAR EDDY-VISCOSITY MODELS}

In this section, we introduce a quantitative measure based on tensorial decomposition to estimate the magnitude of the discrepancy between the eigen-directions of the Reynolds stress tensor based 


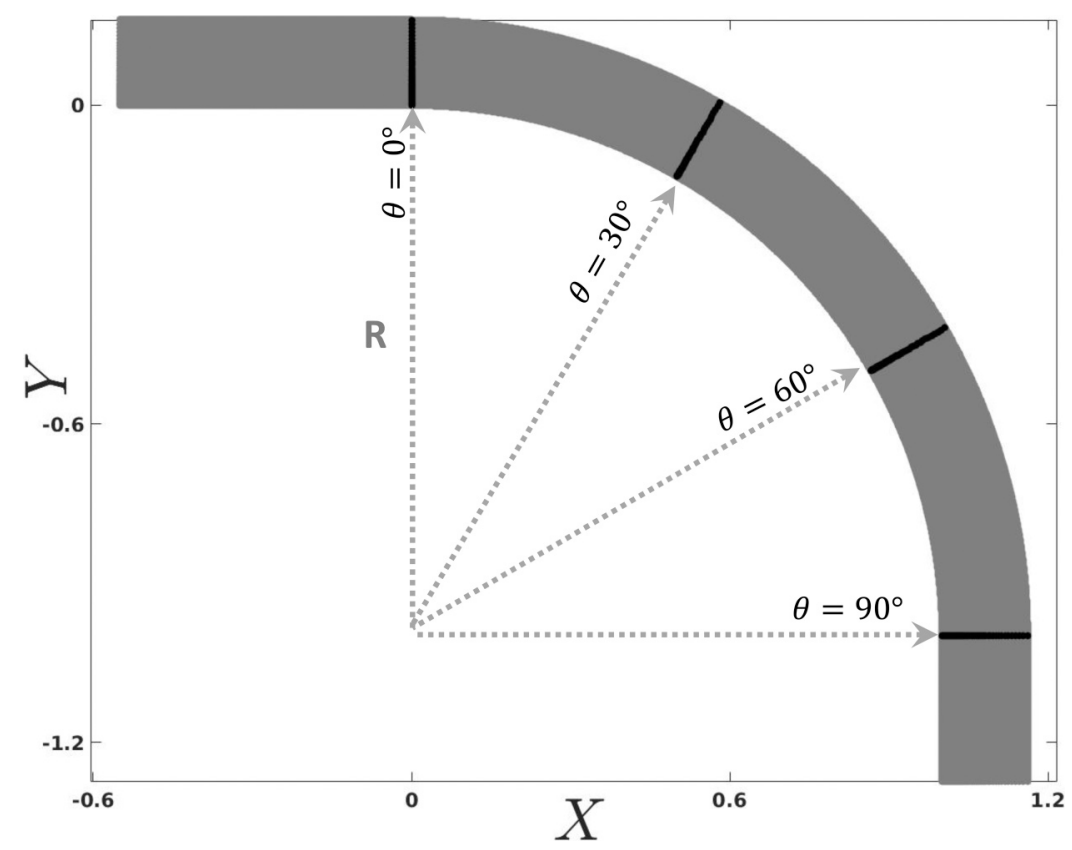

FIG. 2. Flow geometry and profile markers for the convex channel of Arolla and Durbin [24].

on the eddy-viscosity hypothesis as opposed to those from high-fidelity simulations. This is applied to specific cases of turbulent flow over curved surfaces to establish the importance of this eigenvector uncertainty due to RANS models. Turbulent flows over curved surfaces are subjected to streamwise pressure gradients along with additional mean straining. Such curvature can potentially alter flow stability due to the impingement or divergence of the inviscid core $[25,26]$. In such cases, the turbulent flow also becomes complicated due to the manifestation of small scale structures [27]. Linear eddy-viscosity-based models often provide unsatisfactory predictions for such cases and numerous prior investigators have resorted to explicit corrections to capture some of the curvature effects [28-30]. Our test problem is a case of turbulent flow through a convex channel studied in Arolla and Durbin [24]. The computational domain and locations of profiles (where data is available) for the LES study are outlined in Fig. 2. The momentum thickness Reynolds number at the beginning of the curve section is $R_{\theta}=1520$. In the simulation, the straight inlet section is of dimension $14 \delta_{0}$, where $\delta_{0}$ is the $99 \%$ boundary layer thickness. The square cross section has dimension $3 \delta_{0}$. The upper wall is a slip wall, the lower wall is a no-slip wall and the side walls are periodic. In Fig. 2, the circular section, with $R_{\text {inner }}=1$ is delineated using dotted lines. Different sections along this are differentiated using their angle with the beginning of the curved section, as shown in the figure. As an evaluation step, we compute the extent to which the Boussinesq approximation is able to capture the Reynolds stresses computed from the high-fidelity simulations. To this end, we employed the method developed by Thompson et al. [31], i.e., to project the Reynolds stress tensor onto the direction of the rate of strain tensor. We have also projected the Reynolds stress tensor onto an extended basis of mean kinematic quantities. Thompson [32] proposed an index to capture the importance of a tensor (in this case $\mathbf{R}_{\text {mod }}$ ) with respect to a second one (in this case $\mathbf{R}_{\mathbf{H F}}$ ) where the first is an additive part of the second. This scalar index, $\phi$, is given by

$$
\phi=1-\frac{2}{\pi} \cos ^{-1} \frac{\left\|\mathbf{R}_{\text {mod }}\right\|}{\left\|\mathbf{R}_{\mathbf{H F}}\right\|},
$$



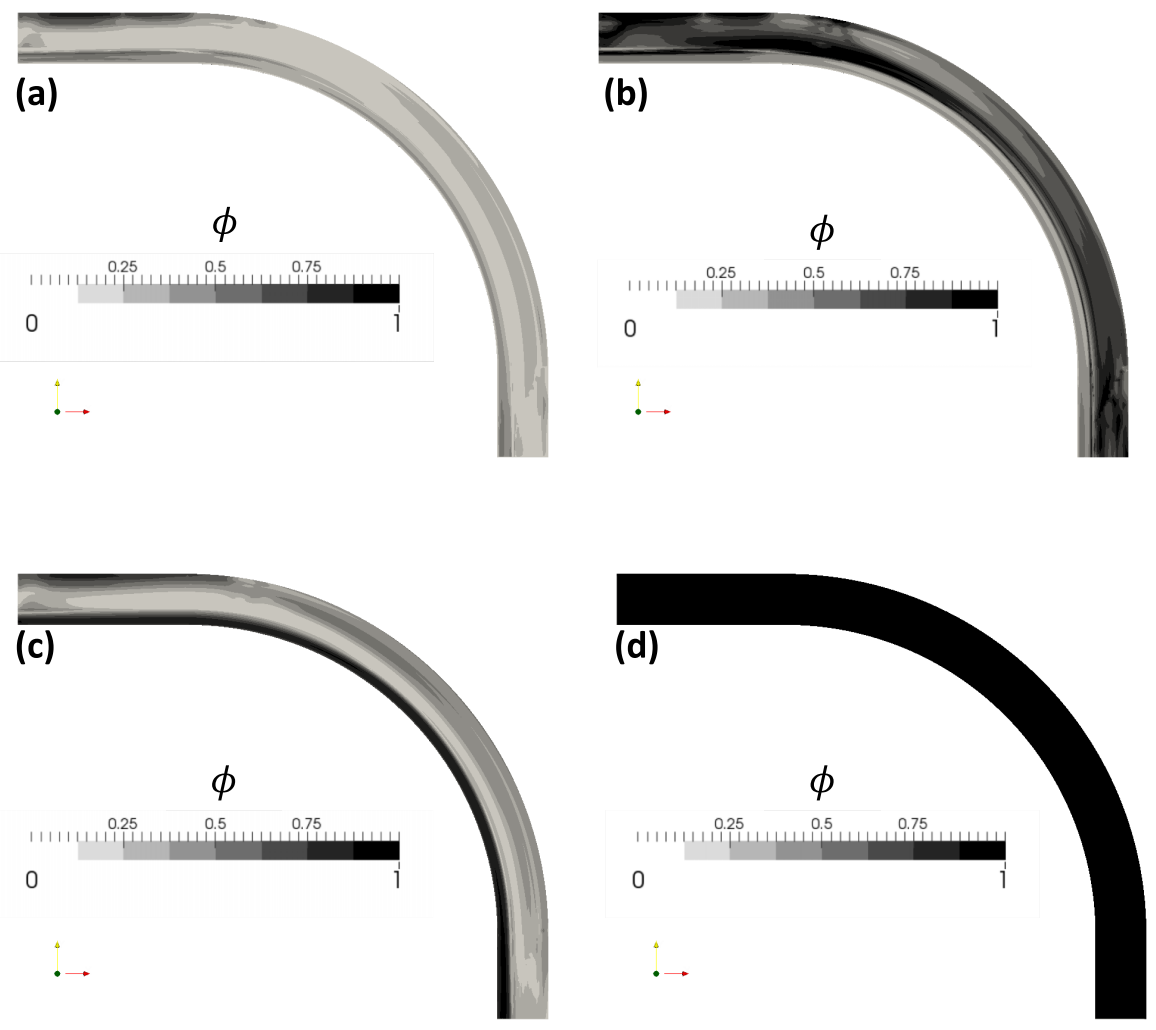

FIG. 3. Indices of performance, $\phi$, associated with the tensor bases considered.

where $\mathbf{R}_{\mathbf{H F}}$ is the Reynolds stress tensor that is obtained from a high-fidelity simulation, $\mathbf{R}_{\mathbf{m o d}}$ is the modeled Reynolds stress tensor, and the symbol $\| .||$ represents the norm, defined as

$$
\|A\|=\sqrt{\sum_{i=1}^{m} \sum_{j=1}^{n} a_{i j}^{2}}
$$

where $a_{i j}$ is an element of $A$. The construction is such that $\mathbf{R}_{\mathbf{H F}}=\mathbf{R}_{\mathbf{m o d}}+\mathbf{E}$, where $\mathbf{E}$ can be seen as a measure of the error associated with the model. This index lies in [0,1], with the limiting cases of $\phi=0$ when the modeled Reynolds stress tensor is uncorrelated with the high-fidelity data, and $\phi=1$, when these are proportional.

This tensorial decomposition and projection enables us to estimate the extent to which the Reynolds stress tensor is coaxial (or shares the same eigen-vectors) with the mean rate of strain. The four cases of the constitutive relation for the Reynolds stress anisotropy, $\mathbf{B}$, that are being evaluated here can be expressed as: Case (a): $\mathbf{B}=\alpha \mathbf{S}$; Case (b): $\mathbf{B}=\alpha_{0} \mathbf{I}+\alpha_{1} \mathbf{S}+\alpha_{2} \mathbf{S}^{\mathbf{2}}$; Case (c): $\mathbf{B}=\alpha_{0} \mathbf{I}+\alpha_{1} \mathbf{S}+\alpha_{2} \mathbf{P}$; and Case d: $\mathbf{B}=\alpha_{0} \mathbf{I}+\alpha_{1} \mathbf{S}+\alpha_{2} \mathbf{S}^{2}+\alpha_{3} \mathbf{P}$. Case (a) represents the classical linear eddy viscosity hypothesis and the latter represent nonlinear eddy-viscosity expressions with an appended tensor basis. These nonlinear eddy-viscosity models are linear combinations of $\mathbf{I}, \mathbf{S}$, and $\mathbf{S}^{2}$ (where $\mathbf{S}$ is the mean rate of strain tensor) and the nonpersistence-of-straining tensor, $\mathbf{P}$ (which represents the local ability of the flow to avoid being persistently stretched [32]). To obtain the efficiency of variants of the eddy-viscosity hypothesis $\left(\mathbf{B}=v_{T} \mathbf{S}\right)$, we decompose $\mathbf{B}$ as [33]

$$
\mathbf{B}=\alpha \mathbf{S}^{\|}+\beta \mathbf{S}^{\perp},
$$


where $\mathbf{S}^{\|}$is the component of $\mathbf{B}$ that is in-phase with $\mathbf{S}$ (sharing the eigen-directions) and thus can be expressed using the linear eddy-viscosity relation. $\mathbf{S}^{\perp}$ represents the component of $\mathbf{B}$ that is orthogonal to $\mathbf{S}$.

Figure 3 shows the performance of the linear eddy viscosity hypothesis, and, the nonlinear eddyviscosity expressions. From Fig. 3(a) we can see that the projection of the RST from the LES data onto the rate-of-strain tensor, is not high with a mean value below 0.5 for the domain. Consequently, the eigen-directions of the high-fidelity predictions of the Reynolds stress anisotropy and the mean rate of strain are not commensurate to a notable degree. The performance fields reveal that adding the nonlinear term $\mathbf{S}$ helps the model prediction far from the wall as observed in Fig. 3(b), whereas the inclusion of tensor $\mathbf{P}$ helps to capture the Reynolds stress tensor near the wall as observed in Fig. 3(c). When both tensors are added, Fig. 3(d), to the usual Boussinesq linear eddy-viscosity model, a negligible error is found. This extended tensor basis has been used in nonlinear eddy viscosity models specifically formulated for flows with curvature [34]. From the figures, it can be observed that modeling only the component of the Reynolds stress anisotropy that is in phase with the mean rate of strain is inadequate to account for the RANS discrepancy. Thus, to account for the out of phase component, a perturbation of the eigendirections of modeled Reynolds stress tensor is critically important.

\section{RESULTS}

In the rest of the article, we outline the results from the application of the proposed eigenvector perturbation methodology, in conjunction with spatially uniform eigenvalue perturbations, to complex turbulent flows. The criteria for evaluation of the results are twofold. First, the results from the proposed methodology should be able to account for a significant proportion of the discrepancy between the unperturbed RANS simulation and the high-fidelity data. Second, the uncertainty intervals using the proposed methodology should exhibit improvement over eigenvalue-only perturbation results, else the additional eigenvector perturbations represent a needless computational burden.

The process of deriving uncertainty intervals from the set of perturbed simulations is schematically reported in Fig. 4. The central row of Fig. 4 outlines the unperturbed, baseline CFD solution, giving a unique flow field realization in the domain and a single profile for the Quantity of Interest in the highlighted region (here, the mean velocity at $x / H=24$ in the diffuser, where $H$ represents half of the height of the diffuser inlet). $x$ and $y$ denote the streamwise and spanwise coordinates. $u_{i}$ is the velocity at the inlet and is used to normalize $u$, the streamwise velocity at a point. The upper and lower panels of the figure outline perturbed solutions (only two of the three perturbations are shown for clarity). Each perturbation leads to a different realization of the flow field, leading

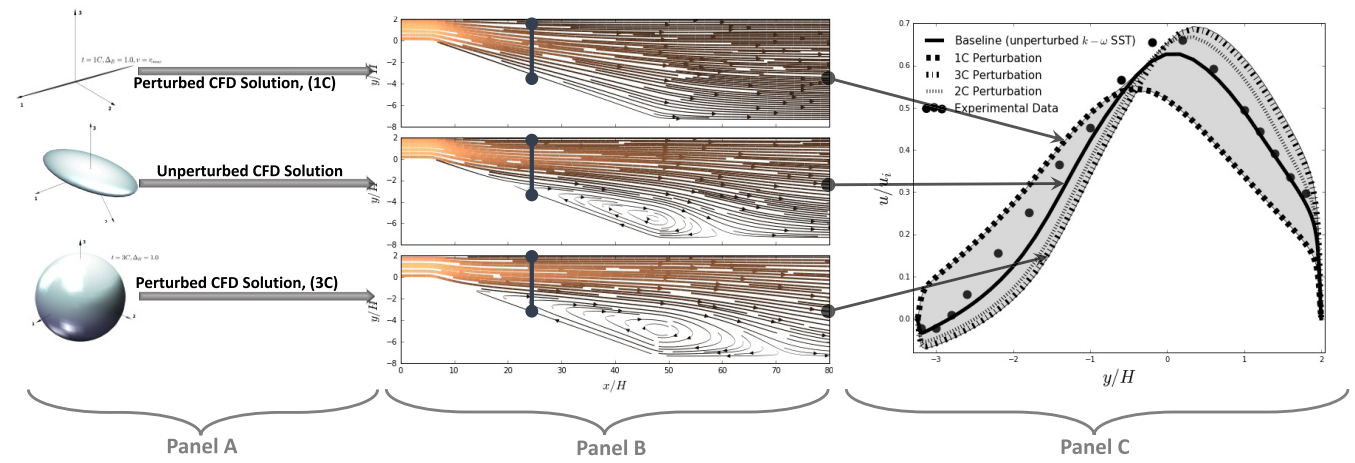

FIG. 4. Schematic outlining the composition of uncertainty estimates for an asymmetric diffuser at $x / H=24$. 
to a different profile for the QoI. The uncertainty estimates on the profiles of a QoI at a location are produced by the union over all the states lying in the profiles from this set of perturbed RANS simulations. This is illustrated by the gray shaded zone in Fig. 4(C). The results presented in this section focus on uncertainty quantification in complex turbulent flows. In all cases, the $k-\omega$ SST model [35] was used and the unperturbed model prediction are reported as the baseline solution. For the eigenvalue perturbations, we utilize the value $\Delta_{B}=1$ for the results in this section. This parameter can be varied if comparative high-fidelity data is available from similar simulations. However, in this investigation we adhere to a physics-based approach and utilize this maximal value of $\Delta_{B}$ to generate conservative estimates. It should be pointed out that the Reynolds stress ellipsoid for the $3 C$ state is a sphere and thus, has complete rotational symmetry. Consequently, if $\Delta_{B}=1$ is chosen for the eigenvalue perturbations, the eigenvector perturbations do not affect this specific perturbed simulation. The effects of variation of this parameter on uncertainty estimates is detailed in Emory et al. [10].

\section{A. Turbulent flow through a convex channel}

The turbulent flow through a convex channel is challenging for RANS closures as the flow is affected by the combined effects of significant streamline curvature along with adverse pressure gradients. Additionally, the mean flow is directly related to the channel geometry. Thus, with the steep change in the channel curvature, sudden changes in mean strain rate occur due to mean streamline curvature. However, these mean flow changes precede any response in the turbulence to the curvature, creating a "lag" between the mean flow and turbulence. These phenomena emphasize the shortcomings of the isotropic eddy viscosity approach and RANS model predictions are of limited fidelity. Edeling et al. [36] have utilized such lag-based models, that can be derived as corrections to the turbulent viscosity assumption, to account for the RANS model uncertainty in such flows.

In Fig. 5, we report a comparison of the $x$-velocity profiles in the curved section of the channel at $0^{\circ}, 30^{\circ}$, and $60^{\circ}$, outlining uncertainty intervals from the proposed eigenvalue-eigenvector perturbation methodology contrasted against uncertainty intervals from eigenvalue-only perturbations. The solid lines represents the predictions of the (unperturbed) $k-\omega$ SST model. The symbols represent the high-fidelity LES data, from the study by Arolla and Durbin [24]. The gray-shaded zones represent the uncertainty intervals. It is seen that eigenvalue only perturbations predict negligibly small uncertainty intervals and do not account for the RANS discrepancy. Primarily, this shortcoming of the eigenvalue perturbation only approach occurs as a significant portion of the RANS discrepancy is due to the misalignment between the eigenvectors of the mean rate of strain and the Reynolds stresses, contrary to what is assumed in isotropic eddy-viscosity models. The proposed eigenvalue-eigenvector perturbation methodology is able to predict substantial uncertainty intervals. While all the LES data is not contained in the interval, a significant proportion of the RANS discrepancy is accounted for.

In Fig. 6, the calculated uncertainty intervals are outlined for the turbulent kinetic energy profiles at $0^{\circ}, 30^{\circ}, 60^{\circ}$, and $90^{\circ}$, marked in Fig. 6(a). The LES data are in agreement with the expectation that the convex curvature reduces turbulence intensity. However, without any added correction terms, the SST model predictions have inadequate sensitivity to streamline curvature. Yang and Tucker [37] have found that even Reynolds stress models are unable to exhibit significant improvement in such convex channel flows, without explicit corrections. In all cases the $k-\omega$ SST model predicts an inordinately high rate of mixing close to the wall. Correspondingly, the maximum value of turbulent kinetic energy in the profile is also underpredicted. However, the predicted uncertainty intervals are able to account for a large proportion of the discrepancy between the RANS predictions and the high-fidelity LES data. The perturbations toward the one-component state form the upper bound of the interval and account for the maximum value of turbulent kinetic energy in the profile. The perturbations toward the three-component state form the lower bound and account for the outer edge of the shear layer. 

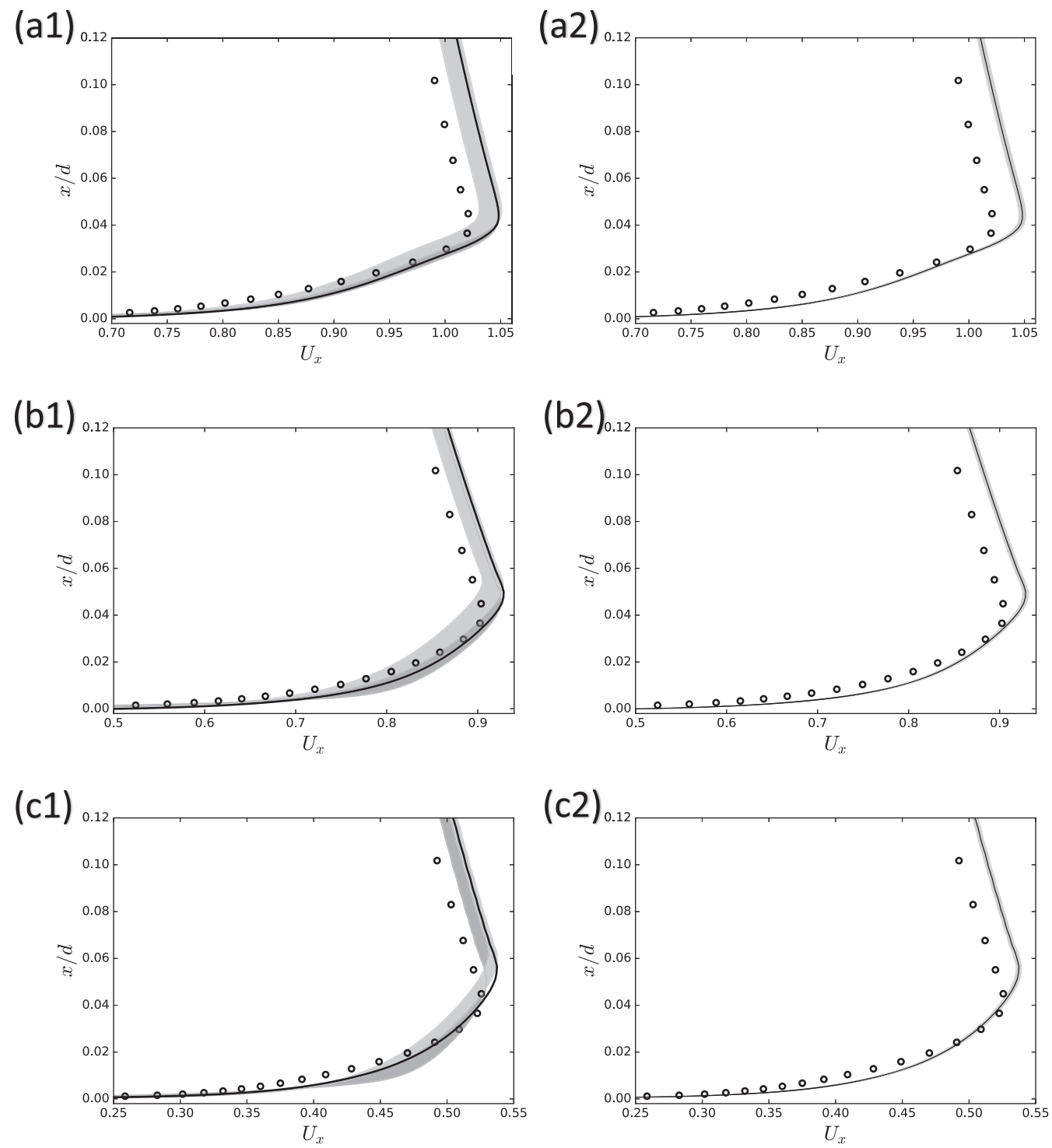

FIG. 5. $X$ velocity $\left(U_{x}\right)$ profiles along the radial direction $x$, with LES comparisons at (a) $0^{\circ}$, (b) $30^{\circ}$, and (c) $60^{\circ}$ locations along the channel. The LES data is represented by circles, the baseline (unperturbed) RANS prediction by the dark line and the uncertainty estimates by the gray-shaded zone. At each location, we exhibit uncertainty intervals from the proposed eigenvalue-eigenvector perturbation methodology contrasted against those from eigenvalue-only perturbations.

\section{B. Turbulent flow through an asymmetric diffuser}

Diffusers are utilized in jet engines, for instance to compress the air flow ahead of turbine engine combustors, and, to slow the air intake to the compressor. Eddy-viscosity-based RANS models have challenges in providing accurate predictions for such flows [38,39]. This discrepancy between RANS predictions and high-fidelity simulations arises primarily due to two separate sources. First, eddy-viscosity-based models are unable to account for the Reynolds stress anisotropy governing the secondary currents in the inflow duct $[40,41]$. This discrepancy in the componentiality of the 

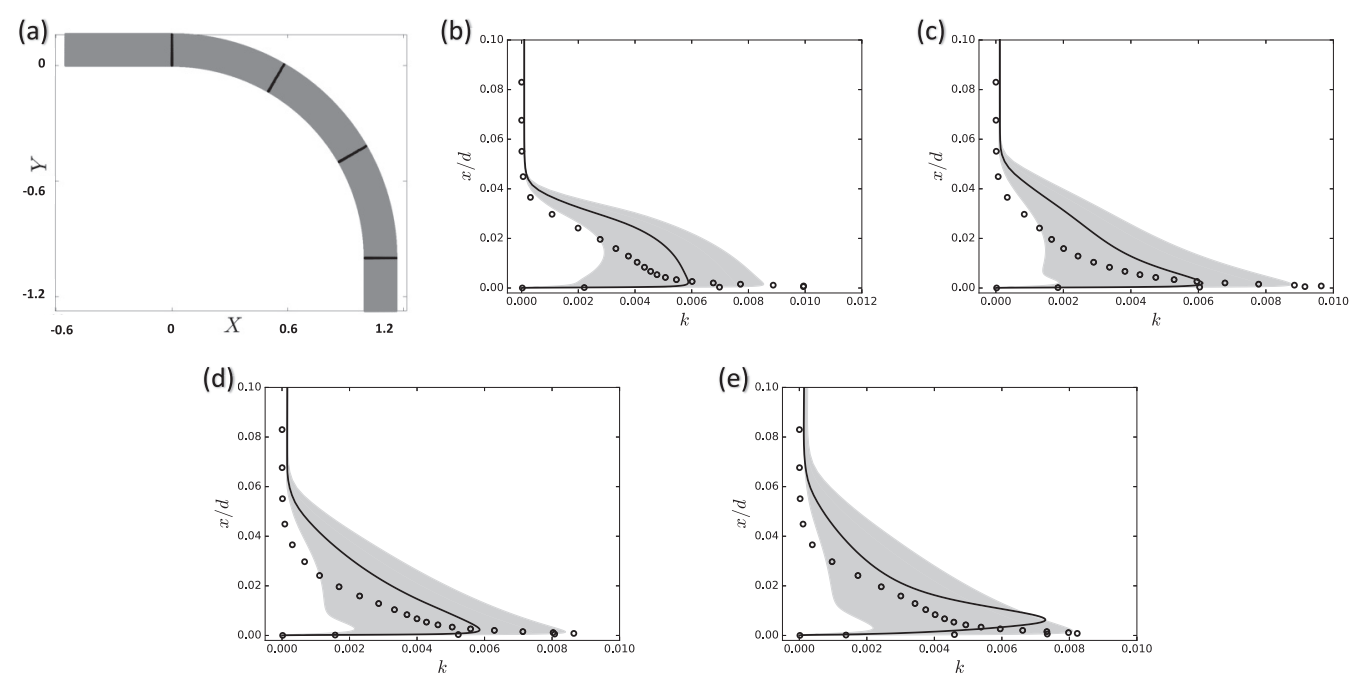

FIG. 6. Turbulent kinetic energy profiles, along with LES comparisons at at (b) $0^{\circ}$, (c) $30^{\circ}$, (d) $60^{\circ}$, and (e) $90^{\circ}$ locations along the channel marked in (a). The LES data is represented by circles, the baseline (unperturbed) RANS prediction by the dark line and the uncertainty estimates by the gray-shaded zone.

inflow to the expansion section necessitates exploring the possible shapes of the Reynolds stress ellipsoid, via eigenvalue perturbations. However, in the diffuser section, the flow also undergoes separation over a smooth wall and ensuing reattachment. Eddy viscosity models assume that the eigendirections of the mean rate of strain are coincident with the Reynolds stress tensor. In regions of flow separation this is an unsatisfactory assumption as there is significant misalignment between the eigendirections of the mean rate of strain and the Reynolds stresses. Thus, accounting for the uncertainty arising due to flow separation requires eigenvector perturbations.

We perform RANS simulations, with eigenvalue and eigenvector perturbations, corresponding to the experiment of Buice and Eaton [42] whose geometry is outlined in Fig. 7, where data for the mean velocity profiles and the friction coefficient are available. Figure 8 shows the coefficient of friction $\left(C_{f}\right)$ over the lower surface of the diffuser, reporting uncertainty intervals from the proposed eigenvalue-eigenvector perturbation methodology contrasted against uncertainty intervals from eigenvalue-only perturbations. The unperturbed, baseline RANS simulation substantially overpredicts the length of the separation bubble. If we restrict ourselves to eigenvalue only perturbations, the uncertainty intervals are negligibly small and do not account for most of the RANS discrepancy. The central cause of RANS discrepancy is due to the flow separation and reattachment at the lower surface of the diffuser. Due to this, the eigenvectors of the mean velocity gradient and the Reynolds stress have significant misalignment, obviating the assumption of the eddy-viscosity models. In this light, eigenvalue only perturbations do not address the discrepancy due to this misalignment and are thus, not of engineering utility. The uncertainty estimate using the proposed eigenvalue-eigenvector

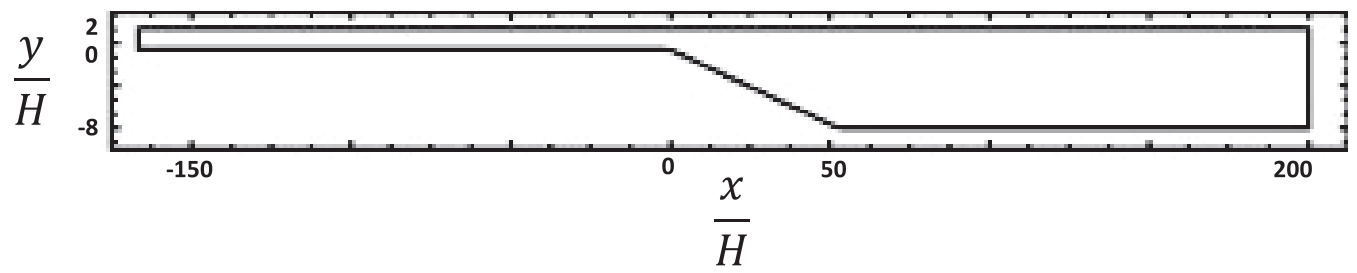

FIG. 7. Flow geometry for the asymmetric diffuser. 

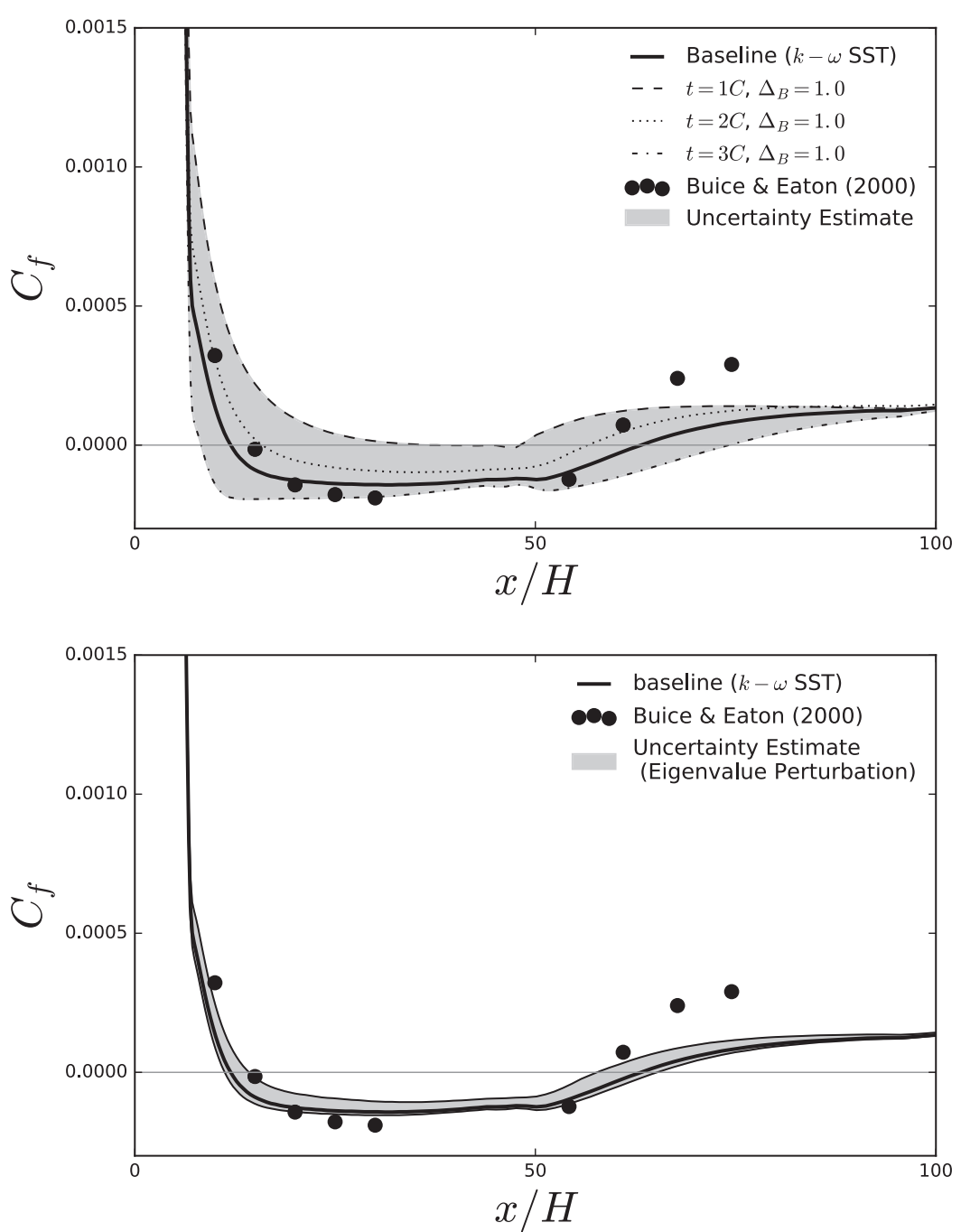

FIG. 8. Coefficient of friction along the bottom wall of the diffuser.

perturbations account for this source of discrepancy and subsume the correct extent of the separation bubble. While the flow separation and reattachment are accounted for by the perturbations, it is unable to account for the ensuing redevelopment of the downstream boundary layer. This causes some of the experimental data after re-attachment to lie outside the uncertainty estimate.

The mean velocity profiles in the expanding section of the diffuser are reported in Fig. 9. The locations are outlined in Fig. 9(a). While there is significant discrepancy between the RANS simulation and the experimental data, the uncertainty estimate is able to account for a significant proportion of this discrepancy, including the separation at the lower surface of the diffuser.

\section{Limitations and future steps}

In the turbulent flows considered, this approach is able to account for a significant proportion of the discrepancy between RANS model predictions and high-fidelity data. However, in some cases, certain high-fidelity data points remain beyond the predicted ranges. This alludes to some limitations in the approach and steps that need to be addressed. We discuss some of these in this section. 

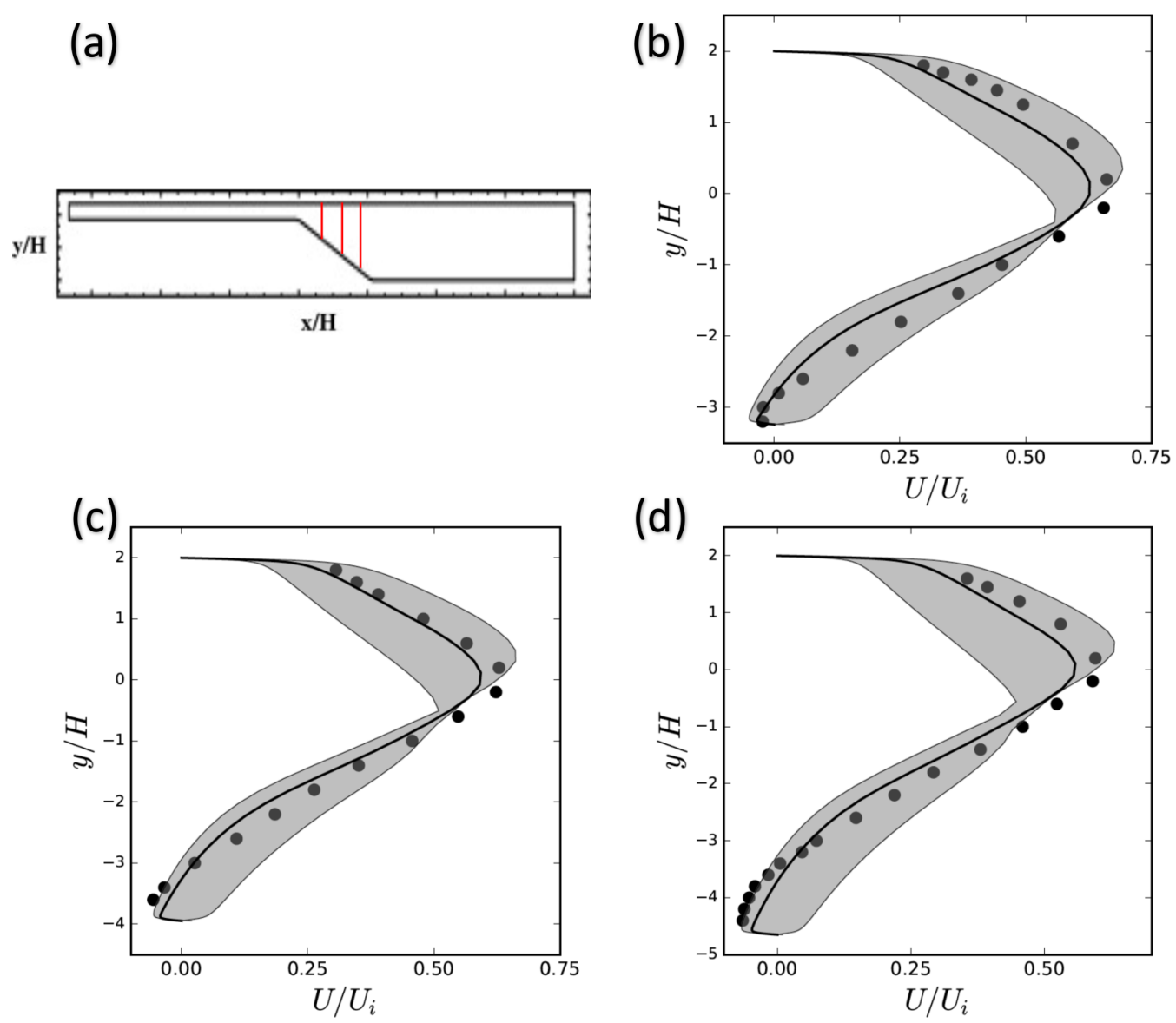

FIG. 9. Mean velocity profiles along the expansion section of the diffuser at locations (b) $x / H=24.4$, (c) $x / H=28.4$, and (d) $x / H=32.4$.

\section{Spatial dependence function}

It is well accepted that RANS model uncertainty is not uniform over flow domains, but is rather more pronounced in specific regions. In this light, the perturbations applied should also be spatially varying to reflect this. In this investigation, we have utilized spatially uniform eigenvalue perturbations. Development of a marker function to guide the spatial variation in the magnitude of perturbations is an important step. This would have a cascading effect on the eigenvector perturbations, which while spatially varying, are still affected by the eigenvalue perturbation.

\section{Turbulent kinetic energy perturbations}

A substantial amount of discrepancy in RANS predictions arises due to the limitations in modeling the turbulent transport process. In RANS models, the turbulent transport is modeled via a gradient-diffusion hypothesis. In the perturbation approach toward uncertainty estimation, the turbulent kinetic energy perturbations are responsible to accounting for this specific limitation [10]. Consequently, results without turbulent kinetic energy perturbations are not able to account for this discrepancy. 


\section{Improvements to eigenvector perturbation approach}

While the results from the eigenvector perturbation approach outlined are encouraging, there are improvements that we are actively addressing. For instance, the computational cost can be reduced by using algebraic Reynolds stress models [43]. Furthermore, the computational procedure is being refined where the $\Delta b_{\text {eval }}$ is divided into smaller steps and corresponding eigenvector perturbations are calculated during this transition, to improve convergence.

\section{CONCLUSIONS}

To establish Reynolds-averaged Navier-Stokes models as a reliable engineering tool, it is required to have dependable estimates of the uncertainties in their predictions. A key source of these RANS uncertainties is due to the structural limitations of such models, including the use of the isotropic eddy-viscosity hypothesis, the gradient-diffusion hypothesis, etc. These structural uncertainties can be quantified using perturbations to the modeled Reynolds stress tensor. These include perturbations to both the eigenvalues and eigenvectors of the Reynolds stresses. While the eigenvalue perturbation methodology is well established, the eigenvector perturbation methodology is still relatively nascent.

In this article, we suggest a new approach to prescribe eigenvector perturbations using a predictor-corrector approach, that uses the incipient eigenvalue perturbations along with the Reynolds stress transport equations to determine the eigenvector perturbations. A key advantage of the proposed methodology is that due to the use of the Reynolds stress transport equations, it accounts for the spatial variation in perturbations. Additionally, the use of the Reynolds stress transport equations leads to "constraining" the eigenvector perturbations within limits. A key hurdle in physics-based perturbation approaches is that they have to rely on the extreme cases to predict bounds. However, the degree of misalignment (between the eigendirections of the mean rate of strain and the Reynolds stresses) that is possible need not be manifested in a turbulent flow. The methodology proposed in this article does not rely on such extreme misalignments and thus, leads to less conservative estimates of the RANS uncertainty.

This methodology was applied to complex turbulent flows where a significant portion of the RANS discrepancy could be attributed to flow separation or streamline curvature. The uncertainty intervals estimated using the proposed framework exhibited substantial improvement over eigenvalue-only perturbations and are able to account for a significant proportion of the discrepancy between RANS predictions and high-fidelity data.

[1] P. E. Hamlington and W. J. A Dahm, Reynolds stress closure for nonequilibrium effects in turbulent flows, Phys. Fluids 20, 115101 (2008).

[2] B. E. Launder, D. P. Tselepidakis, and B. A. Younis, A second-moment closure study of rotating channel flow, J. Fluid Mech. 183, 63 (1987).

[3] C. G. Speziale, T. B. Gatski, and Nessan Mac Giolla Mhuiris, A critical comparison of turbulence models for homogeneous shear flows in a rotating frame, Phys. Fluids A 2, 1678 (1990).

[4] T. J. Craft, B. E. Launder, and K. Suga, Development and application of a cubic eddy-viscosity model of turbulence, Int. J. Heat Fluid Flow 17, 108 (1996).

[5] F.-S. Lien and M. A. Leschziner, Assessment of turbulence-transport models including nonlinear RNG eddy-viscosity formulation and second-moment closure for flow over a backward-facing step, Comput. Fluids 23, 983 (1994).

[6] G. Mompean, S. Gavrilakis, L. Machiels, and M. O. Deville, On predicting the turbulence-induced secondary flows using nonlinear k- $\varepsilon$ models, Phys. Fluids 8, 1856 (1996).

[7] A. P. Singh and K. Duraisamy, Using field inversion to quantify functional errors in turbulence closures, Phys. Fluids 28, 045110 (2016). 
[8] E. J. Parish and K. Duraisamy, A paradigm for data-driven predictive modeling using field inversion and machine learning, J. Comput. Phys. 305, 758 (2016).

[9] J. Ling and J. Templeton, Evaluation of machine-learning algorithms for prediction of regions of high Reynolds-averaged Navier-Stokes uncertainty, Phys. Fluids 27, 085103 (2015).

[10] M. Emory, J. Larsson, and G. Iaccarino, Modeling of structural uncertainties in Reynolds-averaged Navier-Stokes closures, Phys. Fluids 25, 110822 (2013).

[11] G. Iaccarino, A. A. Mishra, and S. Ghili, Eigenspace perturbations for uncertainty estimation of singlepoint turbulence closures, Phys. Rev. Fluids 2, 024605 (2017).

[12] C. Gorlé and G. Iaccarino, A framework for epistemic uncertainty quantification of turbulent scalar flux models for Reynolds-averaged Navier-Stokes simulations, Phys. Fluids 25, 055105 (2013).

[13] C. Gorlé, C. Garcia-Sanchez, and G. Iaccarino, Quantifying inflow and RANS turbulence model form uncertainties for wind engineering flows, J. Wind Eng. Industr. Aerodynam. 144, 202 (2015).

[14] A. A. Mishra, G. Iaccarino, and K. Duraisamy, Epistemic uncertainty in statistical Markovian turbulence models, in Center for Turbulence Research Annual Research Briefs (Stanford University, Stanford, CA, 2016), pp. 183-195.

[15] A. A. Mishra and G. Iaccarino, RANS predictions for high-speed flows using enveloping models, in Center for Turbulence Research Annual Research Briefs (Stanford University, Stanford, CA, 2016), pp. 289-300.

[16] H. Xiao, J.-L. Wu, R. Wang, and C. J. Roy, Quantifying and reducing model form uncertainties in Reynolds-averaged Navier-Stokes simulations: A data-driven, physics-informed approach, J. Comput. Phys. 324, 115 (2016).

[17] J.-L. Wu, J.-X. Wang, and H. Xiao, A Bayesian calibration-prediction method for reducing model-form uncertainties with application in RANS simulations, Flow Turbul. Combust. 97, 761 (2016).

[18] H. Xiao, J.-X. Wang, and R. G. Ghanem, A random matrix approach for quantifying model-form uncertainties in turbulence modeling, Comput. Methods Appl. Mech. Eng. 313, 941 (2017).

[19] J.-L. Wu, H. Xiao, and E. Paterson, Physics-informed machine-learning approach for augmenting turbulence models: A comprehensive framework, Phys. Rev. Fluids 3, 074602 (2018).

[20] J. Wu, R. Sun, S. Laizet, and H. Xiao, Representation of Reynolds stress perturbations with application in machine-learning-assisted turbulence modeling, Comput. Methods Appl. Mech. Eng. 346, 707 (2019).

[21] J.-X. Wang, R. Sun, and H. Xiao, Quantification of uncertainties in turbulence modeling: A comparison of physics-based and random matrix theoretic approaches, Int. J. Heat Fluid Flow 62, 577 (2016).

[22] J.-X. Wang, J. L. Wu, and H. Xiao, Physics-informed machine learning approach for reconstructing Reynolds stress modeling discrepancies based on DNS data, Phys. Rev. Fluids 2, 034603 (2017).

[23] S. Banerjee, R. Krahl, F. Durst, and C. Zenger, Presentation of anisotropy properties of turbulence, invariants versus eigenvalue approaches, J. Turbul. 8, N32 (2007).

[24] S. K. Arolla and P. A. Durbin, LES of spatially developing turbulent boundary layer over a concave surface, J. Turbul. 16, 81 (2015).

[25] P. Bradshaw, The analogy between streamline curvature and buoyancy in turbulent shear flow, J. Fluid Mech. 36, 177 (1969).

[26] J. C. Gillis and J. P. Johnston, Turbulent boundary-layer flow and structure on a convex wall and its redevelopment on a flat wall, J. Fluid Mech. 135, 123 (1983).

[27] W. S. Saric, Görtler vortices, Annu. Rev. Fluid Mech. 26, 379 (1994).

[28] P. R. Spalart and M. Shur, On the sensitization of turbulence models to rotation and curvature, Aerosp. Sci. Technol. 1, 297 (1997).

[29] D. C. Wilcox and T. L. Chambers, Streamline curvature effects on turbulent boundary layers, AIAA J. 15, 574 (1977).

[30] S. K. Arolla and P. A. Durbin, Modeling rotation and curvature effects within scalar eddy viscosity model framework, Int. J. Heat Fluid Flow 39, 78 (2013).

[31] R. L. Thompson, G. Mompean, and L. Thais, A methodology to quantify the nonlinearity of the Reynolds stress tensor, J. Turbul. 11, N33 (2010).

[32] R. L. Thompson, Some perspectives on the dynamic history of a material element, Int. J. Eng. Sci. 46, 224 (2008). 
[33] T. G. Kolda, Orthogonal tensor decompositions, SIAM J. Matrix Anal. Appl. 23, 243 (2001).

[34] C. L. Rumsey, T. B. Gatski, and J. H. Morrison, Turbulence model predictions of strongly curved flow in a u-duct, AIAA J. 38, 1394 (2000).

[35] F. R. Menter, Two-equation eddy-viscosity turbulence models for engineering applications, AIAA J. 32, 1598 (1994).

[36] W. N. Edeling, G. Iaccarino, and P. Cinnella, Data-free and data-driven RANS predictions with quantified uncertainty, Flow Turbul. Combust. 100, 593 (2018).

[37] X. Yang and P. G. Tucker, Assessment of turbulence model performance: Large streamline curvature and integral length scales, Comput. Fluids 126, 91 (2016).

[38] E. M. Cherry, C. J. Elkins, and J. K. Eaton, Geometric sensitivity of three-dimensional separated flows, Int. J. Heat Fluid Flow 29, 803 (2008).

[39] E. M. Cherry, G. Iaccarino, C. J. Elkins, and J. K. Eaton, Separated flow in a three-dimensional diffuser: Preliminary validation, in Center for Turbulence Research Annual Research Briefs (Stanford University, Stanford, CA, 2006), pp. 57-83.

[40] H. Steiner, S. Jakirlic, G. Kadavelil, R. Manceau, S. Saric, and G. Brenn, 13th ERCOFTAC workshop on refined turbulence modelling, ERCOFTAC Bull. 78, 22 (2009).

[41] S. Jakirlic, G. Kadavelil, S. Sirbubalo, D. von Terzi, M. Breuer, and D. Borello, SIG15 workshop on refined turbulence modelling, ERCOFTAC Bull. 85, 5 (2010).

[42] C. U. Buice and J. K. Eaton, Experimental investigation of flow through an asymmetric planar diffuser, Report TSD-107, Department of Mechanical Engineering (Stanford University, Stanford, CA, 1997).

[43] S. Wallin and A. V. Johansson, An explicit algebraic Reynolds stress model for incompressible and compressible turbulent flows, J. Fluid Mech. 403, 89 (2000). 